\title{
Discrete Burr Type II Distribution
}

\author{
Dr. Nahed Helmy \\ Faculty of Commerce-Al-Azhar University \\ Girls' Branch \\ Egypt
}

Abstract

This paper is concerned with introducing discrete Burr Type II distribution. The non-centrally moments, central moments, the skewness and the kurtosis measures are obtained. Reliability, hazard rate, quantile, mode and median are derived. Distribution of the smallest, the largest order statistics, the lower record value and the upper record value are obtained. Parameter estimation using maximum likelihood method and Bayesian approach is obtained. To illustrate the proposed model, numerical example was given.

Key words: Lower record and upper record values, Newton Raphson, Order Statistics, Maximum likelihood estimation, Bayesian estimation, Burr Type II distribution.

\section{Introduction}

Burr distributions have numerous applications. For example, in problems related to hypothesis testing, lifetime data, statistical quality control, in survival and reliability studies. In addition, it is employed in financial studies, economics, income and wage distribution as well as in environmental studies.

Roy (2003) pointed out that the normal distribution is playing a key role in stochastic modeling for a continuous setup. But its distribution function does not have an analytical form. For this requirement they proposed a discrete 
version of the continuous normal distribution. Characterization results are made to establish a direct link between the discrete normal distribution and its continuous counterpart. The corresponding concept of a discrete approximator for the normal deviate are suggested. An application of the discrete normal distributions for evaluating the reliability of complex systems is elaborated as an alternative to simulation methods.

Krishna and Pundir (2009) obtained discrete Burr and Pareto distributions using the general approach of discretizing a continuous distribution and proposed them as suitable lifetime models. Some important distributional and estimation of reliability characteristics are discussed. An application in reliability estimation in series system and a real data example on dentistry using this distribution is also discussed.

Jazi et al. (2010) proposed a discrete inverse Weibull distribution. They showed that the hazard rate function can attain a unimodal or monotone decreasing shape for certain values of parameters. They studied four methods of estimation (the heuristic algorithm, the inverse Weibull probability paper plot, the method of moments and the method of proportions). From the results of extensive simulation runs, their accuracies and precisions are compared. It is found that for right skewed discrete inverse Weibull distributions, the last two methods seem wanting due to certain characteristics of the estimation procedures and numerical convergence. The inverse Weibull probability paper plot and the heuristic method fare better. Finally, a discrete data set is fitted by both the discrete Weibull and the discrete inverse Weibull and their AICs are compared.

Gómez-Déniz and Calderín-Ojeda (2011) introduced a new probability mass function by discretizing the continuous failure model of the Lindley distribution. The model obtained is over-dispersed and competitive with the Poisson distribution to fit automobile claim frequency data. After revising some of its properties a compound discrete Lindley distribution is obtained in closed form. This model is suitable to be applied in the collective risk model when both number of claims and size of a single claim are implemented into the model. The new compound distribution fades away to zero much more slowly than the classical compound Poisson distribution, being therefore suitable for modelling extreme data. 
AL-Huniti and AL-Dayian (2012) introduced the discrete Burr Type III distribution using the general approach of discretizing a continuous distribution and proposed it as a suitable lifetime model. The equivalence of continuous and discrete Burr Type III distribution is established. Some important distributional properties and estimation of the parameters, reliability, failure rate and the second rate of failure functions are discussed based on the maximum likelihood method and Bayesian approach.

Chakraborty and Chakravarty (2012) derived a two-parameter discrete gamma distribution which is corresponding to the continuous two parameters gamma distribution using the general approach for discretization of continuous probability distributions. One parameter discrete gamma distribution is obtained as a particular case. A few important distributional and reliability properties of the proposed distribution are examined. Parameter estimation by different methods is discussed. Performance of different estimation methods are compared through simulation. Data fitting is carried out to investigate the suitability of the proposed distribution in modeling discrete. Failure time data and other count data.

Nekoukhou et al. (2015) introduced a discrete analogue of beta exponential distribution which is more plausible in modeling discrete data and exhibits both increasing and decreasing hazard rates. They studied some basic distributional and moment properties of the new distribution. Then certain structural properties of the distribution such as its unimodality, hazard rate behavior and Renyi entropy are discussed. Using the maximum likelihood method estimation of the model parameters is also investigated. The model is examined with areal data set and compared with its rival model that is the discrete generalized exponential distribution.

Para and Jan (2016) proposed a discrete three parameter Burr Type XII distribution and discrete Lomax distribution as new discrete models using the general approach of discretization of continuous distribution. They studied some basic distributional and moment properties of these new distributions. Then, certain structural properties of the distributions such as their unimodality, hazard rate behaviors and the second rate of failure functions are discussed. Various theorems relating a three-parameter discrete Burr Type XII distribution and discrete Lomax distribution with other statistical distributions 
are proved. They are examined the models with data set of counts of cysts of kidneys using steroids and compared with the classical models.

Oliveira et al. (2017) studied an alternative discrete Lindley distribution to those study in G'omez-D'enize Calder'1n-Ojeda (2011) and Bakouch et al. (2014). For both distributions, a simulation study is carried out to examine the bias and mean squared error for the maximum likelihood estimators of the parameters as well as the coverage probability and the width of the confidence intervals. For the discrete Lindley distribution obtained by infinite series method they presented the analytical expression for bias reduction of the maximum likelihood estimator. Some examples using real data from the literature showed the potential of these distributions. Despite the discretization methods are quite different, the resulting distributions are interchangeable, however the distribution generated by an infinite series has simple mathematical expressions and can be used directly to count data in the presence of covariates.

Para and Jan (2017) introduced a new count data model which is obtained by compounding discrete Burr Type XII distribution with Minimax distribution. Several distributional properties of the model are discussed. Real data set is analyzed to investigate the suitability of the proposed distribution in modeling count.

This paper is divided into five sections. The first section is the introduction. The second section contains discretizing the continuous distribution. The third section is devoted to the model of the discrete Burr Type II distribution.Section four contains the estimation of the parameter. Section five contains the numerical study.

\section{Discretizing a Continuous Distribution}

A continuous failure time model can be used to generate a discrete model by introducing a grouping on the time axis. If the underlying continuous failure time $\mathrm{X}$ has the reliability function (RF), $R(x)=p(X \geq x)$ and times are grouped into unit intervals so that the discrete observed variable is $d X=$ $[X]$ the largest integer part of $X$ the probability mass function (pmf) of $d X$ can be written as 


$$
\begin{gathered}
p(x)=p[d X=x]=p(x \leq d X \leq x+1)=R(x)-R(x+1), X \\
=0,1,2, \ldots
\end{gathered}
$$

The $p m f$ of discrete random variable $(d X)$ can be viewed as discrete concentration of the $p d f$ of $X$. The first and easiest in this approach is the geometric distribution with $p m f$

$p(x)=\theta^{x}(1-\theta)=\theta^{x}-\theta^{x+1}, X=0,1,2, \ldots$

This is obtained by discretizing the exponential distribution with RF

$R(x)=e^{-\lambda x}=\theta^{x}, \lambda, x>0$ Here $\theta=e^{-\lambda},(0<\theta<1) \rightarrow R(x)=\theta^{x}$

\section{The Model of the Discrete Burr Type II Distribution}

\subsection{Continuous Burr Type II Distribution}

A lifetime $r v X$ follows the Burr Type $I I$ distribution Burr $I I(k)$ if its $p d f$ is given by

$f(x)=k e^{-x}\left(1+e^{-x}\right)^{-(k+1)},-\infty<x<\infty, k>0$

and its cumulative distribution function is given by

$F(x)=\left(1+e^{-x}\right)^{-k},-\infty<x<\infty, k>0$

The corresponding reliability function $(R F)$ failure rate function $(H R F)$ are respectively given by

$$
\begin{aligned}
& R(x)=1-\left(1+e^{-x}\right)^{-k},-\infty<x<\infty, k>0 \\
& h(x)=\frac{f(x)}{R(x)}=\frac{k e^{-x}\left(1+e^{-x}\right)^{-(k+1)}}{1-\left(1+e^{-x}\right)^{-k}},-\infty<x<\infty, k>0
\end{aligned}
$$

The plots of the $p d f(x)$ and $h(x)$ are provided in Figures (1) and (2) respectively 


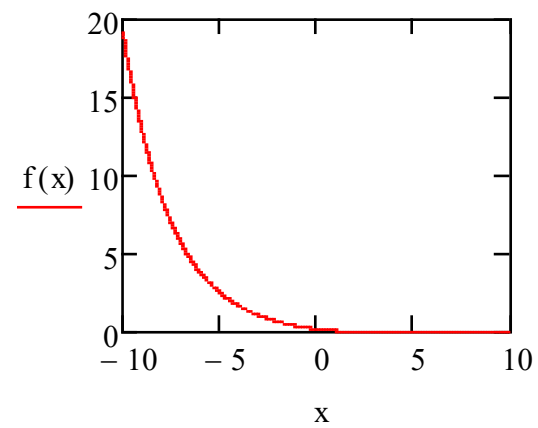

Figure (1) $p d f(x)$ for $d B u r r I I$ distribution at $\theta=1.5$

From Figure (1) it is noticed that $p d f(x)$ is approximately has exponential distribution.

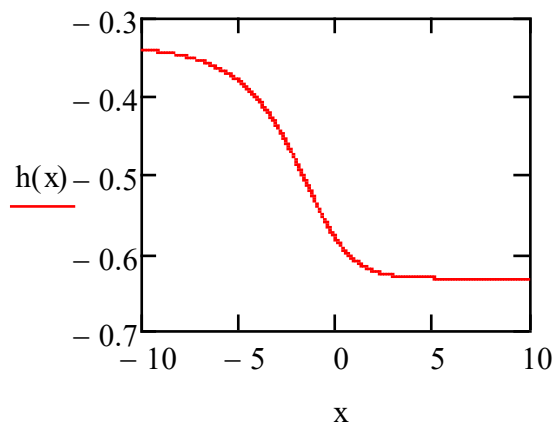

Figure (2) $h(x)$ for $d B u r r ~ I I$ at $\theta=1.5$

From Figure (2) it is noticed that $h(x)$ for $d B u r r$ II distribution is decreased then it becomes constant.

\subsection{Discrete Burr Type II Distribution:}

Based on the reliability function of continuous Burr II $r v X$ which is given by (5) the $R(x)$ for $d B u r r I I(k, \theta)$ distribution at integer point of $X$ is given by:

$R(x)=1-\theta^{\log \left(1+e^{-x}\right)}$

where $\theta=e^{-k}$ and $(0<\theta<1)$ 
Now by using (1) the pmf of the discrete Burr Type $I I$ distribution with parameter $\theta d B$ urr $I I(\theta)$ can be defined as:

$$
\begin{aligned}
& p(x)=R(x)-R(x+1)=\theta^{\log \left(1+e^{-(x+1)}\right)}-\theta^{\log \left(1+e^{-x}\right)}, \\
& x=-\infty, \ldots, 0,1,2, \ldots
\end{aligned}
$$

and the cumulative distribution function is given by:

$$
\begin{aligned}
& F(x)=\theta^{\log \left(1+e^{-x}\right)}, \\
& x=-\infty, \ldots, 0,1,2, \ldots
\end{aligned}
$$

The $r t h$ non-centrally moments of $d B u r r I I(\theta)$ is given by:

$$
\begin{aligned}
\mu_{r}^{\prime}=E\left(X^{r}\right)= & \sum_{x=0}^{\infty} x^{r} p(x) \\
& =\sum_{x=0}^{\infty} x^{r}\left[\theta^{\log \left(1+e^{-(x+1)}\right)}-\theta^{\log \left(1+e^{-x}\right)}\right]
\end{aligned}
$$

- The mean of lifetime $\mu$ of $d B u r r I I(\theta)$ can be obtained by using (9) as follows:

$\dot{\mu}_{1}=\mu=\sum_{x=0}^{\infty} x\left[\theta^{\log \left(1+e^{-(x+1)}\right)}-\theta^{\log \left(1+e^{-x}\right)}\right]$

- the second non-centrally moment is given by:

$$
\dot{\mu}_{2}=\sum_{x=0}^{\infty} x^{2}\left[\theta^{\log \left(1+e^{-(x+1)}\right)}-\theta^{\log \left(1+e^{-x}\right)}\right]
$$

- The $3^{\text {rd }}$ non-centrally moment is given by:

$$
\dot{\mu}_{3}=\sum_{x=0}^{\infty} x^{3}\left[\theta^{\log \left(1+e^{-(x+1)}\right)}-\theta^{\log \left(1+e^{-x}\right)}\right]
$$

- The $4^{\text {th }}$ non-centrally moment is given by: 


$$
\dot{\mu}_{4}=\sum_{x=0}^{\infty} x^{4}\left[\theta^{\log \left(1+e^{-(x+1)}\right)}-\theta^{\log \left(1+e^{-x}\right)}\right]
$$

The central moments of $d B u r r I I(\theta)$ are given by:

- The variance $V(\theta)$ of $d B u r r I I(\theta)$ can be obtained by using Eqs. (10) and (11) as follows:

$$
\begin{aligned}
& V(\theta)=\mu_{2}-\mu^{2} \\
& =\sum_{x=0}^{\infty} x^{2}\left[\theta^{\log \left(1+e^{-(x+1)}\right)}-\theta^{\log \left(1+e^{-x}\right)}\right] \\
& -\left[\sum_{x=0}^{\infty} x\left[\theta^{\log \left(1+e^{-(x+1)}\right)}-\theta^{\log \left(1+e^{-x}\right)}\right]\right]^{2}
\end{aligned}
$$

- The $3^{\text {rd }}$ centrally moment is given by:

$\mu_{3}=\mu_{3}-2 \mu_{2}^{\prime} \mu+\mu^{3}=\sum_{x=0}^{\infty} x^{3}\left[\theta^{\log \left(1+e^{-(x+1)}\right)}-\theta^{\log \left(1+e^{-x}\right)}\right]-$

$2 \sum_{x=0}^{\infty} x^{2}\left[\theta^{\log \left(1+e^{-(x+1)}\right)}-\theta^{\log \left(1+e^{-x}\right)}\right]\left\{\sum_{x=0}^{\infty} \mathrm{x}\left[\theta^{\log \left(1+e^{-(x+1)}\right)}-\theta^{\log \left(1+e^{-x}\right)}\right]\right\}$

$+\left[\sum_{x=0}^{\infty} \mathrm{x}\left[\theta^{\log \left(1+e^{-(x+1)}\right)}-\theta^{\log \left(1+e^{-x}\right)}\right]\right]^{3}$

- The $4^{\text {th }}$ centrally moment is given by:

$$
\begin{aligned}
& \mu_{4}=\mu_{4}^{\prime}-4 \mu_{3}^{\prime} \mu+6 \mu_{2} \mu^{2}-3 \mu^{4}=\sum_{x=0}^{\infty} x^{4}\left[\theta^{\log \left(1+e^{-(x+1)}\right)}-\theta^{\log \left(1+e^{-x}\right)}\right] \\
& -4\left\{\sum_{x=0}^{\infty} x^{3}\left[\theta^{\log \left(1+e^{-(x+1)}\right)}-\theta^{\log \left(1+e^{-x}\right)}\right]\right\}\left\{\sum_{x=0}^{\infty} \mathrm{x}\left[\theta^{\log \left(1+e^{-(x+1)}\right)}-\theta^{\log \left(1+e^{-x}\right)}\right]\right\}
\end{aligned}
$$




$$
\begin{aligned}
& +6\left\{\sum_{x=0}^{\infty} x^{2}\left[\theta^{\log \left(1+e^{-(x+1)}\right)}-\theta^{\log \left(1+e^{-x}\right)}\right]\right\}\left\{\sum_{x=0}^{\infty} \mathrm{x}\left[\theta^{\log \left(1+e^{-(x+1)}\right)}-\theta^{\log \left(1+e^{-x}\right)}\right]\right\}^{2} \\
& -3\left\{\sum_{x=0}^{\infty} \mathrm{x}\left[\theta^{\log \left(1+e^{-(x+1)}\right)}-\theta^{\log \left(1+e^{-x}\right)}\right]\right\}^{4}
\end{aligned}
$$

- The skewness measure $\alpha_{3}$ of $d B u r r I I(\theta)$ can be obtained by using Eqs. (10), (11) and (12) as follows:

$$
\begin{aligned}
& \alpha_{3}=\frac{\mu_{3}-2 \mu_{2} \mu+\mu^{3}}{V^{3 / 2}} \\
& =\frac{\sum_{x=0}^{\infty} x^{3}\left[\theta^{\log \left(1+e^{-(x+1)}\right)}-\theta^{\log \left(1+e^{-x}\right)}\right]-2 \sum_{x=0}^{\infty} x^{2}\left[\theta^{\log \left(1+e^{-(x+1)}\right)}-\theta^{\log \left(1+e^{-x}\right)}\right]}{\left[\sum_{x=0}^{\infty} x^{2}\left[\theta^{\log \left(1+e^{-(x+1)}\right)}-\theta^{\log \left(1+e^{-x}\right)}\right]-\left[\sum_{x=0}^{\infty} x\left[\theta^{\log \left(1+e^{-(x+1)}\right)}-\theta^{\log \left(1+e^{-x}\right)}\right]\right]^{2}\right]^{\frac{3}{2}}} \\
& =\frac{\sum_{x=0}^{\infty} x\left[\theta^{\log \left(1+e^{-(x+1)}\right)}-\theta^{\log \left(1+e^{-x}\right)}\right]+\left[\sum_{x=0}^{\infty} x\left[\theta^{\log \left(1+e^{-(x+1)}\right)}-\theta^{\log \left(1+e^{-x}\right)}\right]\right]^{3}}{\left[\sum_{x=0}^{\infty} x^{2}\left[\theta^{\log \left(1+e^{-(x+1)}\right)}-\theta^{\log \left(1+e^{-x}\right)}\right]-\left[\sum_{x=0}^{\infty} x\left[\theta^{\log \left(1+e^{-(x+1)}\right)}-\theta^{\log \left(1+e^{-x}\right)}\right]\right]^{2}\right]^{\frac{3}{2}}}
\end{aligned}
$$

- The kurtosis $\alpha_{4}$ of $d B u r r I I(\theta)$ can be obtained by using Eqs. (10), (11), (12), and (13) as follows:

$$
\begin{aligned}
& \alpha_{4}=\frac{\mu_{4}-4 \mu_{3} \mu+6 \mu_{2} \mu^{2}-3 \mu^{4}}{\mu_{2}^{2}} \\
& =\frac{\sum_{x=0}^{\infty} x^{4}\left[\theta^{\log \left(1+e^{-(x+1)}\right)}-\theta^{\log \left(1+e^{-x}\right)}\right]-4\left\{\sum_{x=0}^{\infty} x^{3}\left[\theta^{\log \left(1+e^{-(x+1)}\right)}-\theta^{\log \left(1+e^{-x}\right)}\right]\right\}}{\left[\sum_{x=0}^{\infty} x^{2}\left[\theta^{\log \left(1+e^{-(x+1)}\right)}-\theta^{\log \left(1+e^{-x}\right)}\right]-\left[\sum_{x=0}^{\infty} x\left[\theta^{\log \left(1+e^{-(x+1)}\right)}-\theta^{\log \left(1+e^{-x}\right)}\right]\right]^{2}\right]^{2}} \\
& =\frac{\left\{\sum_{x=0}^{\infty} \mathrm{x}\left[\theta^{\log \left(1+e^{-(x+1)}\right)}-\theta^{\log \left(1+e^{-x}\right)}\right]\right\}+6\left\{\sum_{x=0}^{\infty} x^{2}\left[\theta^{\log \left(1+e^{-(x+1)}\right)}-\theta^{\log \left(1+e^{-x}\right)}\right]\right\}}{\left[\sum_{x=0}^{\infty} x^{2}\left[\theta^{\log \left(1+e^{-(x+1)}\right)}-\theta^{\log \left(1+e^{-x}\right)}\right]-\left[\sum_{x=0}^{\infty} x\left[\theta^{\log \left(1+e^{-(x+1)}\right)}-\theta^{\log \left(1+e^{-x}\right)}\right]\right]^{2}\right]^{2}}
\end{aligned}
$$

$$
\frac{\left\{\sum_{x=0}^{\infty} \mathrm{x}\left[\theta^{\log \left(1+e^{-(x+1)}\right)}-\theta^{\log \left(1+e^{-x}\right)}\right]\right\}^{2}-3\left\{\sum_{x=0}^{\infty} \mathrm{x}\left[\theta^{\log \left(1+e^{-(x+1)}\right)}-\theta^{\log \left(1+e^{-x}\right)}\right]\right\}^{4}}{\sum_{x=0}^{\infty} x^{2}\left[\theta^{\log \left(1+e^{-(x+1)}\right)}-\theta^{\log \left(1+e^{-x}\right)}\right]-\left[\sum_{x=0}^{\infty} x\left[\theta^{\log \left(1+e^{-(x+1)}\right)}-\theta^{\log \left(1+e^{-x}\right)}\right]\right]^{2}}
$$




\subsection{Quantile function is:}

$F(q)=p \rightarrow F\left[\theta^{\log \left(1+e^{-q}\right)}\right]=\mathrm{p}$

It can be shown from the previous equation that the $q^{\text {th }}$ quantile function for the $d B u r r I I(\theta)$ distribution is:

$q=\ln \left(\frac{1}{p-1-\theta}\right)$

at $q=0.5$ the median of $d B u r r I I(\theta)$ distribution is obtained.

- We have shown that the mode of the random variable $X$ using the recurrence relation is:

mode $=\frac{\theta^{\log \left(1+e^{-(x+2)}\right)}-\theta^{\log \left(1+e^{-(x+1)}\right)}}{\theta^{\log \left(1+e^{-(x+1)}\right)}-\theta^{\log \left(1+e^{-x}\right)}}$

Let $x_{1}, x_{2}, \ldots, x_{n}$ be any real valued random variables and its ordered values denoted as $x_{(1)} \leq X \leq \cdots \leq x_{(n)}$ then the values $x_{(1)} \leq x_{(2)} \leq \cdots \leq x_{(n)}$ are the order statistics of the random variable, $X$.

It can be shown that, the $p d f$ of the $r^{\text {th }}$ order statistic is given by:

$$
\begin{aligned}
& \cdot f_{(r)}\left(x_{(r)}\right)=\frac{n !}{(r-1) !(n-r) !}\left[F ( x _ { ( r ) } ] ^ { r - 1 } \left[1-F\left(x_{(r)}\right]^{n-r} f\left(x_{(r)}\right)\right.\right. \\
& =\frac{n !}{(r-1) !(n-r) !}\left[\theta^{\log \left(1+e^{-x}(r)\right)}\right]^{r-1}\left[1-\theta^{\log \left(1+e^{-x}(r)\right)}\right] * \\
& {\left[\theta^{\log \left(1+e^{-\left(x(r)^{+1)}\right.}\right)}-\theta^{\log \left(1+e^{-x}(r)\right)}\right]}
\end{aligned}
$$

It can be shown that the density of the $n^{\text {th }}$ ordered statistics follows the $d B u r r I I(\theta)$ distribution is as follows:

$$
\begin{aligned}
& f_{(n)}\left(x_{(n)}\right)=n\left[F\left(x_{(n)}\right]^{n-1} f\left(x_{(n)}\right)\right. \\
= & n\left[\theta^{\log \left(1+e^{-x}(n)\right)}\right]^{n-1}\left[\theta^{\log \left(1+e^{-\left(x_{(n)}+1\right)}\right)}-\theta^{\log \left(1+e^{-x}(n)\right)}\right]
\end{aligned}
$$


Suppose that the smallest value follows the $\operatorname{dBurr} \operatorname{II}(\theta)$ distribution then it can be shown that the density of the smallest order statistic is given by:

$$
\begin{gathered}
-f_{(1)}\left(x_{(1)}\right)=n\left[1-F\left(x_{(1)}\right]^{n-1} f\left(x_{(1)}\right)\right. \\
=n\left[1-\theta^{\log \left(1+e^{-x}(1)\right)}\right]^{n-1}\left[\theta^{\log \left(1+e^{-\left(x_{(1)}+1\right)}\right)}-\theta^{\log \left(1+e^{\left.-x_{(1)}\right)}\right.}\right]
\end{gathered}
$$

It can be shown that the upper record value is:

$$
\begin{gathered}
\text { - } f_{U(n)}(x)=\frac{1}{\Gamma(n)}[-\log (1-F(x))]^{n-1} f(x) \\
\left.\left.=\frac{1}{\Gamma(n)}\left[1-\log \left(\theta^{\log \left(1+e^{-x} u(n)\right.}\right)\right)\right]^{n-1}\left[\theta^{\log \left(1+e^{-(x u(n)+1)}\right)}-\theta^{\log \left(1+e^{-x} u(n)\right.}\right)\right]
\end{gathered}
$$

It can be shown that the lower record value is:

$$
\begin{aligned}
\text { - } f_{L(n)}(x)=\frac{1}{\Gamma(n)}[-\log (F(x))]^{n-1} f(x) \\
=\frac{1}{\Gamma(n)}\left[-\log \left(-\theta^{\log \left(1+e^{-x_{l(n)}}\right)}\right)\right]^{n-1}\left[\theta^{\log \left(1+e^{-\left(x_{l(n)^{+1)}}\right)}-\theta^{\log \left(1+e^{\left.-x_{l(n)}\right)}\right.}\right]}\right.
\end{aligned}
$$

Suppose that $U$ is the standard uniform variate. It can be shown that the random variable $X$ :

$X=\ln \left(\frac{1}{U-1-\theta}\right)$

follows the $d \operatorname{Burr} I I(\theta)$ distribution and is used to generate the random variable for the distribution assuming $\theta$ is known.

\section{Estimation of the Parameter of $\operatorname{dBurr} I I(\theta)$ :}

\subsection{Estimation of the parameter based on the ML method}

The maximum likelihood method is used to estimate the parameter of the $\operatorname{dBurr} I I(\theta)$ distribution. Let $\left(x_{1}, x_{2}, \ldots, x_{n}\right)$ be a random sample from dBurr $I I(\theta)$ distribution with density function as $f(\underline{x} ; \theta)$. The likelihood function of the $d B u r r I I(\theta)$ distribution for the parameter $\theta$ is given as 


$$
\begin{aligned}
L \underline{\underline{x}} \underline{\theta})=\prod_{i=1}^{n} f & (\underline{x} ; \theta) \\
& =\prod_{i=1}^{n}\left[\theta^{\log \left(1+e^{-(x+1)}\right)}-\theta^{\log \left(1+e^{-x}\right)}\right]
\end{aligned}
$$

$L(\underline{x} ; \theta)=\prod_{i=1}^{n}\left[\theta^{\log \left(1+e^{-x}\right)}\left[\theta^{\log \left(\frac{1+e^{-(x+1)}}{1+e^{-x}}\right)}-1\right]\right]$

where $\phi\left(x_{i}\right)=\log \left(\frac{1+e^{-(x+1)}}{1+e^{-x}}\right)$

and

$\log L(x ; \theta)=\sum_{i=1}^{n}\left\{\log \left(1+e^{-(x+1)}\right) \log \theta+\log \left[\theta^{\phi\left(x_{i}\right)}-1\right]\right\}$

Partially differentiating (29) with respect to the parameter $\theta$ we have the following equation

$\frac{\partial \log L}{\partial \theta}=\sum_{i=1}^{n}\left[\frac{\log \left(1+e^{-(x+1)}\right)}{\widehat{\theta}}+\frac{\phi\left(x_{i}\right) \widehat{\theta}^{\phi\left(x_{i}\right)-1}}{\widehat{\theta}^{\phi\left(x_{i}\right)}-1}\right]=0$

The maximum likelihood estimate (MLEs) of $\theta$ can be obtained by solving (30) numerically.

\subsection{Bayesian Estimation:}

The probability density function $(p d f)$ and cumulative distribution function $(c d f)$ of the discrete Burr Type $I I$ distribution are given respectively as:

$$
\begin{aligned}
& f(x, \theta)=\theta^{\log \left(1+e^{-(x+1)}\right)}-\theta^{\log \left(1+e^{-x}\right)},-\infty<X<\infty ; \theta>0 \\
& F(x, \theta)=\theta^{\log \left(1+e^{-x}\right)},-\infty<X<\infty ; \theta>0
\end{aligned}
$$

where $\theta$ is the shape parameter.

\subsubsection{Loss Functions and Posterior Distribution}

We will use the following loss functions:

1. The squared error loss function (SELF) is a symmetric loss function and takes the form:

$L(\theta, \hat{\theta})=c(\theta-\hat{\theta})^{2}$ 
where $c$ denotes a constant and $\hat{\theta}$ is an estimator. The Bayes estimator with respect to a quadratic loss function is the mean of the posterior distribution which takes the form:

$$
\hat{\theta}_{B}=E(\theta \mid \underline{X})=\int_{\theta} \theta \pi(\theta \mid \mathrm{x}) d \theta=\int_{0}^{\infty} \theta \pi(\theta \mid x) d \theta
$$

2. Calabria and Pulcini (1996) presented a general entropy (GE) loss function when it appears to be realistic to express the loss in terms of the ratio $\left(\frac{\widehat{\theta}}{\theta}\right)$ and defined it as:

$$
L(\theta, \widehat{\theta})=\left(\frac{\hat{\theta}}{\theta}\right)^{q}-\mathrm{q} \log \left(\frac{\hat{\theta}}{\theta}\right)-1
$$

whose minimum occurs at $\hat{\theta}=\theta$. This loss function is a generalization of the entropy loss used by several authors where the shape parameter $q=1$ [Dey and Liu (1992)]. When $q>0$ a positive error $(\hat{\theta}>\theta)$ causes serious consequences than a negative error. The Bayes estimator $\hat{\theta}_{G E}$ of $\theta$ under GE loss is

$$
\hat{\theta}_{G E}=\left[E_{\theta}\left(\theta^{-q}\right)\right]^{-1 / q}=\int_{0}^{\infty} \theta^{-1} \pi(\theta \mid x) d \theta
$$

Provided that the expectation $\left[E_{\theta}\left(\theta^{-q}\right)\right]^{-1 / q}$ exists and finite where $E_{\theta}$ denotes the expected value with respect to the posterior function of $\theta$

\section{The posterior distribution:}

The likelihood function can be obtained as:

$$
L(\theta \mid \underline{X})=\prod_{i=1}^{n}\left[\theta^{\log \left(1+e^{-(x+1)}\right)}-\theta^{\log \left(1+e^{-x}\right)}\right]
$$

We can use the gamma distribution as a conjugate prior distribution of $\theta$ with parameters $a$ and $\mathrm{b}$ as:

$$
\pi(\theta \mid a, b)=\frac{b^{a}}{\Gamma(\mathrm{a})} \theta^{a-1} e^{-b \theta}, \theta>0, a, b>0
$$


where $\Gamma(a)=\int_{0}^{\infty} t^{a-1} e^{-t} d t$ is Gamma function, hyper parameters $a>$ $0, b>0$

The posterior distribution of $\theta$ can be obtained by combining Eqs. (36) and (37) to be:

$$
\begin{aligned}
& \pi(\theta \mid x)=L(\theta \mid \underline{X}) \pi(\theta \mid a, b)=\left(\frac{b^{a}}{\Gamma(\mathrm{a})} \theta^{a-1} e^{-b \theta}\right) \\
& \prod_{i=1}^{n}\left[\theta^{\log \left(1+e^{-(x+1)}\right)}-\theta^{\log \left(1+e^{-x}\right)}\right],(\theta, a, b)>0
\end{aligned}
$$

The Bayes estimator with respect to the quadratic loss function is the mean of the posterior distribution as follows:

$$
\begin{aligned}
& E(\theta \mid x)=\int_{0}^{\infty} \theta \pi(\theta \mid x) d \theta \\
& =\int_{0}^{\infty} \theta\left(\frac{b^{a}}{\Gamma(\mathrm{a})} \theta^{a-1} e^{-b \theta}\right) \prod_{i=1}^{n}\left[\theta^{\log \left(1+e^{-(x+1)}\right)}-\theta^{\log \left(1+e^{-x}\right)}\right] d \theta
\end{aligned}
$$

The Bayes estimator with respect to the GELF loss function is the mean of the posterior distribution as follows:

$$
\begin{aligned}
& \hat{\theta}_{G e}=\left[E_{\theta}\left(\theta^{-q}\right)\right]^{-1 / q}=\int_{0}^{\infty} \theta^{-1} \pi(\theta \mid x) d \theta \\
& =\int_{0}^{\infty} \theta^{-1}\left(\frac{b^{a}}{\Gamma(\mathrm{a})} \theta^{a-1} e^{-b \theta}\right) \prod_{i=1}^{n}\left[\theta^{\log \left(1+e^{-(x+1)}\right)}-\theta^{\log \left(1+e^{-x}\right)}\right] d \theta \quad(q=1)
\end{aligned}
$$

\section{Numerical Study}

\subsection{Monte Carlo Simulation using MLE's}

Some numerical results based on the MLE's of $d B u r r ~ I I(\theta)$ distribution is obtained according the following steps:

1) Given initial value $\theta_{0}$ generate random samples of size $(n=30,100)$ from the $d \operatorname{Burr} I I(\theta)$ by observing that if $\mathrm{U}$ is uniform $(0,1)$ then 
$X=\ln \left(\frac{1}{U-1-\theta}\right)$

which has the discrete Burr Type II distribution.

2) For each sample size $n$ and the initial value of shape parameter $(\theta)$ the MLE's of the parameter $\theta$ can be obtained by solving the nonlinear equation (30) numerically using Mathcad iteration scheme

3) Repeat steps (1) - (2) $\mathrm{m}$ times where $\mathrm{m}=1000$ for $n=30,100$

4) The estimate and mean square error of the MLE's of the parameter $(\theta)$ are computed by averaging over the $m$ repetitions

The computation results are displayed in Table (1)

For the estimated parameter $\theta$ we assume that the actual population value is $\theta=0.4$.

Table (1) display the estimation of the parameter, and the estimated mean square error of the maximum likelihood estimate of $\theta$ for different sample sizes. In this section MLE's of the shape parameter, $\theta$ of the discrete Burr Type II distribution is obtained. MLE'S is found by solving the nonlinear equation (30).

Table (1) the estimate and mean square error of the MLE's of the parameter $(\theta=0.4)$ at $n=30,100)$

\begin{tabular}{|c|c|c|c|}
\hline$n$ & parameter & estimation & MSE \\
\hline 30 & $\theta$ & 0.298 & 0.045 \\
\hline 100 & $\theta$ & 0.302 & 0.042 \\
\hline
\end{tabular}

\section{Concluding Remarks}

It is noticed that the estimated mean square error is decreased as the sample size increased.

\subsection{Monte Carlo Simulation using Bayesian approach}

In this section a Monte Carlo Simulation is performed to assess the performance of the Bayesian estimate associated to the shape parameter $\theta$ of the discrete Burr Type $I I$ distribution discussed in the previous sections. The simulation structure can be described in the following steps: 
1. Set the true value of $\theta$ at $(\theta=0.05)$. We considered different sample sizes $(n=30,100)$ to study their effect on the resulting estimates.

2. For given values of $\left(a_{0}=2, b_{0}=2\right)$ we generate $\theta$ from gamma distribution.

3. Calculate Bayes estimates of the unknown shape parameter associated to the discrete Burr Type II distribution according to the formulas that have been obtained.

4. We repeated this process 1000 times and compute the Mean Square Error (MSE) for the estimate where $\operatorname{MSE}(\hat{\theta})=\frac{1}{1000} \sum_{i=1}^{1000}\left(\hat{\theta}_{i}-\right.$ $\theta)^{2}$ where $\hat{\theta}$ is the estimate of $\theta$. The simulation results are displayed in Table 2 .

The results of Bayesian estimation are displayed in Tables (2), (3).

Table (2): Bayes, estimates based on (SELF) for $(m=$ $1000, n=30,100, \theta=0.05$ )

\begin{tabular}{|c|c|c|c|}
\hline$n$ & parameter & estimation & MSE \\
\hline 30 & $\theta$ & $2.708 \times 10^{-4}$ & $2.524 \times 10^{-3}$ \\
\hline 100 & $\theta$ & $1.116 \times 10^{-5}$ & $2.499 \times 10^{-3}$ \\
\hline
\end{tabular}

From Table (2) it is noticed that the estimated mean square is decreased as the sample size increased.

Table (3): Bayes, estimates based on (Entropyf) for

$$
(m=1000, n=30,100, \theta=0.05)
$$

\begin{tabular}{|c|c|c|c|}
\hline$n$ & parameter & estimation & MSE \\
\hline 30 & $\theta$ & $4.597 \times 10^{-7}$ & $2.5 \times 10^{-3}$ \\
\hline 100 & $\theta$ & $1.124 \times 10^{-7}$ & $2.5 \times 10^{-3}$ \\
\hline
\end{tabular}

From Table (3) it is noticed that the estimated mean square is decreased as the sample size increased. 
It is noticed that the MSE of the shape parameter $\theta$ under the entropy function is less than the one under the squared error loss function when $n=30$ but the MSE of the shape parameter $\theta$ under the entropy function is larger than the one under the squared error loss function when $n=100$.

\subsection{An application using real data}

This application is given by Bhaumik et al. (2009). The data refers to the vinyl chloride data obtained from clean upgradient monitoring wells in $\mathrm{mg} / \mathrm{l}$ :

\begin{tabular}{|c|c|c|c|c|c|c|c|c|}
\hline 5.1 & 1.2 & 1.3 & 0.6 & 0.5 & 2.4 & 0.5 & 1.1 & 8 \\
\hline 0.4 & 2 & 0.5 & 5.3 & 3.2 & 2.7 & 2.9 & 2.5 & 2.3 \\
\hline 1.8 & 0.9 & 2 & 4 & 6.8 & 1.2 & 0.4 & 0.2 & 0.8 \\
\hline 0.6 & 0.1 & 0.9 & 0.1 & 1 & 0.4 & 0.2 & & \\
\hline
\end{tabular}

Using the one-sample Kolmogorov - Smirnov test for goodness of fit the $p-$ value $=0.001$ which is less than the significance level $\alpha=0.01$ so the null hypothesis is rejected and the alternative hypothesis cannot be rejected so these real data has discrete Butt Type II distribution.

The descriptive statistics for these data are obtained in Table (4).

Table (4) Descriptive Statistics for the real data of glass fibers

\begin{tabular}{|c|c|c|c|c|c|c|}
\hline $\mathrm{N}$ & $\min$ & $\max$ & range & mean & S.D. & $Q_{1}$ \\
\hline 34 & 0.1 & 8 & 7.9 & 1.88 & 1.95 & 0.5 \\
\hline$Q_{2}$ & $Q_{3}$ & Skewness & Kurtosis & $\begin{array}{c}\text { Lower } \\
\text { bound }\end{array}$ & $\begin{array}{c}\text { Upper } \\
\text { bound }\end{array}$ & \multicolumn{1}{|}{} \\
\cline { 1 - 5 } 1.15 & 2.55 & 1.68 & 2.54 & 1.19 & 2.56 & \multicolumn{1}{|c}{} \\
\cline { 1 - 4 } & & &
\end{tabular}


Since the value of the skewness measure is positive. So, the distribution of the data is skewed to the right. Also, since the value of the kurtosis measure is less than 3 , so the distribution for the data is flat.

The estimate of the parameter $\theta$ using both MLE's and Bayesian approach is displayed in Table (5).

\section{Table (5) Bayes estimates}

\begin{tabular}{|c|c|}
\hline \multirow{3}{*}{$\hat{\theta}=0.004$ using MLE's } & $\begin{array}{c}\hat{\theta}=0.017 \text { under }(S E L F) \text { using Bayesian } \\
\text { approach }\end{array}$ \\
\cline { 2 - 2 } & $\begin{array}{c}\hat{\theta}=1.484 \times 10^{-7} \text { under }(\text { Entropy } F) \\
\text { using Bayesian approach }\end{array}$ \\
\hline
\end{tabular}

From Table (5) it is noticed that the estimate of the shape parameter $\theta$ under the entropy function is less than the one under the squared error loss function using the Bayesian approach and the one obtained using the ML method.

\section{References}

1. AL-Huniti, A. A. and AL-Dayian, G. R. (2012). "Discrete Burr Type III Distribution." American Journal of Mathematics and Statistics, Vol. 2, pp. 145-152.

2. Bakouch, H. S., Jazi, M. A. and Nadarajah, S. (2014). “A New Discrete Distribution", Statistics, Vol. 48, pp. $200-240$.

3. Calabria, R. and Pulcini, G. (1996). Point estimation under asymmetric loss

function for left truncated exponential samples. Communications in Statistics. Theory and Methods, Vol. 25, pp. 285-600.

4. Chakraborty, S. and Chakravarty, D. (2012). "Discrete gamma Distributions: Properties and Parameter Estimations", 
Communications in Statistics - Theory and Methods, Vol. 41, pp. 3301 -3324 .

5. Gomez-Deniz, E. and Calderin-Ojeda, E. (2011)." The Discrete Lindley Distribution: Properties and Applications." Journal of Statistical Computation and Simulation, Vol. 81, pp. 1405-1416.

6. Jazi, M. A., Lai, C. D. and Alamatsaz, M. H. (2010). "A Discrete Inverse Weibull Distribution and Estimation of its Parameters." Statistical Methodology, Vol. 7, pp. 121-132.

7. Krishna, H. and Pundir, P. S. (2009). "Discrete Burr and Discrete Pareto Distributions.”, Statistical Methodology, Vol. 6, pp. 177-188.

8. Murthy, D. N. P., Xie, M. and Jiang, R. (2004). Weibull Models. Wiley series in probability and statistics, John Wiley and Sons, Inc., New York.

9. Nadarajaj, S. and Kotz,S.(2006). " On the Laplace Transform of the Pareto Distribution", Applied Mathematics

10. Nekoukhou, V., Alamatsaz, M. H., Bidram, H. and Aghajani, A.H. (2015). "Discrete Beta - Exponential Distribution." Communication in Statistics - Theory and methods, Vol. 44, pp. 2079-2091.

11. Oliveira, R. P., Mazucheli, J. and Achcar, J. A. (2017). "A comparative Study between two Discrete Lindley Distributions.", Ciência e Natura, Vol. 39, pp. 539-552.

12. Para, B. A. and Jan, T.R. (2014). "Discrete Generalized Burr Type XII Distribution", Journal of Modern Applied Statistical Methods, Vol. 13, pp. $244-258$. 
13. Para, B. A and Jan. T. R. (2016). "On Discrete Three Parameter Burr Type XII and Discrete Lomax Distributions and Their applications to Model Count Data from Medical Science.", Biometrics and Biostatistics International Journal, Vol. 4, pp. 1-15.

14. Para, B. A. and Jan, T. R. (2017). "Discrete Burr Type XII Minimax Distribution: A New Discrete Model." Journal of Statistics Applications and Probability, Vol. 6, pp. 635-646.

15. Roy, D. (2003). "The Discrete Normal Distribution." Journal of Communications in Statistics - Theory and Methods, Vol. 32, pp. 1871-1883. 


\section{ملخص بالعربي}

هذا البحث يهتم بتقديم توزيع Burr المنفصل من النوع الثاني وقد نم اشتقاق العزوم اللامركزية و المركزية وكل من مقياس الالتو اءو التقرطح وكذلك تم اشتقاق دالة البقاء ودالة الفتل لهذا التوزيع وأيضا تم اشتقاق الوسبط والمنوال. ونم اشتقاق التوزيع الاحتمالي لكل من اصغر قيمة واكبر قيمة من الاحصاءات المرتبة وتم تقدير معلمة التوزيع باستخدام طريقة الامكان الأكبر وطريقة بييز وتم استخدام بيانات فعلية لتوضيح هذا التوزيع 